\title{
Carlos II de España, «El hechizado»
}

\section{Jaime Cerda L. \\ Charles I/ of Spain, «the bewitched»}

The death of King Charles II, the Bewitched», ended two centuries of sovereignity of the Habsburg dynasty in Spain. Since his birth in 1661, he presented a peculiar set of physical, psychiatric and behavioral signs, such as respiratory and diarrheal diseases, recurrent seizures and deep developmental delay. It was not until his adulthood when his infertility became evident, being incapable of conceiving a heir, even though he married twice. Such a constellation of ominous signs motivated a curious investigation, which concluded that the king was hexed at the age of 14 years in order to take away his throne, his health and his capacity to procreate. Based on contemporary medical knowledge, it is possible that Charles II had a rare autosomal recessive inherited genopathy, as a consequence of the frequent inbreeding among his ancestors. On the other hand, its is also possible that Charles II presented Klinefelter Syndrome, the most frequent sex chromosome disorder in humans and the most common cause of hypogonadism and infertility in males. The hypothesis that Charles II was bewitched reflects a deep belief in supernatural phenomena among the Castilian society at the beginning of the $18^{\text {th }}$ century, an idea transmitted across generations, currently present in many societies worldwide (Rev Méd Chile 2008; 136: 263-6).

(Key words: Genetic diseases, inborn; Klinefelter syndrome; Witchcraft)

Recibido el 4 de julio, 2007. Aceptado el 31 de agosto, 2007.

Programa de Especialidad en Salud Pública, Departamento de Salud Pública, Facultad de Medicina, Pontificia Universidad Católica de Chile.

\section{RESEÑA HISTÓRICA}

$\mathrm{E}^{1}$ $1^{0}$ de noviembre de 1700, a los 39 años de edad, falleció el rey Carlos II, último monarca español de la Casa de los Austrias. Su muerte sin sucesión directa puso fin a la dinastía Habsburgo española, iniciada en 1516 por su tatarabuelo Carlos V, Sacro Emperador Romano y nieto de los Reyes Católicos, dando origen a la Guerra de Sucesión Española. Tras el término de este conflicto, se confirmó en el trono a Felipe V, iniciándose la dinastía borbónica española, presente hasta el día de hoy ${ }^{1}$.

Carlos II (Figura 1) fue el quinto hijo del rey Felipe IV y de la archiduquesa Mariana de Austria, su sobrina, ambos retratados en Las Meninas», de

Correspondencia a: Dr. Jaime Cerda L. Departamento de Salud Pública, Facultad de Medicina, Pontificia Universidad Católica de Chile. Marcoleta 434. Santiago, Chile. Tel.: (56 2) 354 3014. Fax: (56 2) 6331840.

E mail: jcerda@med.puc.cl
Velásquez. Su nacimiento, ocumido el día 6 de noviembre de 1661, fue comentado en la «azeta de Madrid», la cual anunció la llegada al mundo de eun robusto varón, de hermosísimas facciones, cabeza

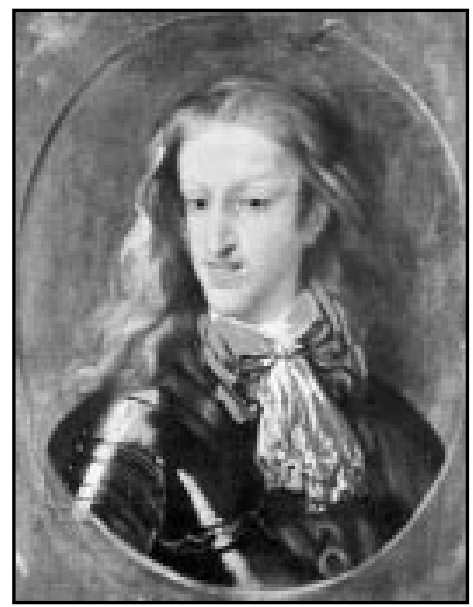

Figura 1. Retrato de Carlos II de España, «el Hechizado» (1661-1700). Disponible en www.answers.com 
proponcionada, pelo negro y algo abultado de carnes». Aquella descripción distaba bastante de la realidad, siendo diametralmente opuesta la impresión que tuvo el embajador de Francia, quien comunicaba a los pocos días los pormenores a Luis XIV, el Rey Sol. En palabras del embajador: el púncipe parece bastante débil; muestra signos de degeneración; tiene flemones en las mejillas, la cabeza llena de costras y el cuello le supura» y más adelante, sasusta de feo». Catorce amas de cría amamantaron a Carlos II hasta los cuatro años, sin embargo, esta forma de alimentación fue suspendida tras su ascenso al trono en 1665, pues era considerada indecorosa para un monarca. El pequeño Carlos creció como un niño débil y enfermizo, tenía frecuentes catarmos, diameas y escasa musculatura y presentaba un evidente retraso en su desarrollo psicomotor, pues cumplidos los seis años aún no había aprendido a caminar. En su historia médica figuran sarampión y varicela a los 6 años, rubéola a los 10 años y viruela a los 11 años; además, el joven rey sufrió ataques epilépticos hasta los 15 años. Sin embargo, lo más llamativo era su escaso desarmollo intelectual, pues tan sólo a los 10 años comenzó a hablar de manera inteligible y nunca aprendió a escribir comectamente; presentaba arranques de cólera imprevisibles y una adicción monoalimentaria al chocolate.

Su mala salud presagiaba que moriría joven, por lo que se descuidó su educación, al punto que nadie se preocupó de prepararle adecuadamente para las tareas de gobierno. Carlos II contaba apenas cuatro años cuando fue coronado rey, siendo la regencia administrada por su madre, asesorada por una junta de gobierno. El testamento de su padre decretaba que su heredero debía ser declarado mayor de edad a los 14 años, sin embargo, su retraso físico y mental era tal, que su madre consiguió un aplazamiento por dos años más. Una vez en el trono, su período como monarca fue agónico para España, reino que sufría por el hambre y por la pérdida de poder y prestigio en el contexto europeo ${ }^{1-3}$.

El problema de la sucesión monárquica fue abordado tempranamente, pues a la edad de 18 años, luego de una fructífera negociación, Carlos II contrajo nupcias con Mańa Luisa de Oréans (1662-1689), una joven de 17 años, sobrina de Luis XIV, el Rey Sol. La boda se celebró el 18 de noviembre de 1679 en el pequeño pueblo de Quintanapalla, cerca de Burgos. Transcumido un año de matrimonio, Mańa Luisa no daba a luz a un heredero, más aún, seguía vírgen, pues el matrimonio nunca se consumó, seguramente debido al hipogonadismo e hipogenitalismo que padecía Carlos. Transcumidos diez años de matrimonio, Mańa Luisa falleció a consecuencia de una apendicitis aguda (su autopsia evidenció un útero indemne), sin haber dado a luz un heredero para la corona, para desgracia de la familia real ${ }^{1-3}$.

Con tan sólo 28 años de edad, la alicaída salud de Carlos II era una constante, siempre débil y fatigado, siendo frecuente sus problemas gastrointestinales, atribuidos a su glotonería y problemas de masticación secundarios a un significativo prognatismo, propio y caracteństico de los Habsburgo ${ }^{4}$. Al cabo de un año de la muerte de Mańa Luisa, la creciente preocupación por la sucesión monárquica motivó la realización de un segundo matrimonio, esta vez con Mariana de Neoburgo. La boda real se celebró en Valladolid el 4 de mayo de 1690, sin embargo, a pesar de su fértil antecedente (los padres de Mariana tuvieron 23 hijos), la descendencia no llegaba. En su desazón, potenciada por las reiteradas simulaciones de embarazo por parte de Mariana, el mismo Carlos sospechaba que un hechizo proferido contra él le impedía engendrar, razón por la cual ordenó en 1698 investigar el tema en profundidad. Los peritajes concluyeron que el rey había sido víctima de un hechizo, el cual se lo habían dado en una taza de chocolate el 3 de abril de 1675, en la que habían disuelto sesos de un ajusticiado para quitarle el gobiemo; entrañas para quitarle la salud y ninones para cormomperle el semen e impedir la generación» Carlos II fue exorcizado mediante una serie de pócimas repugnantes, las cuales sólo lograron empeorar su delicada condición ${ }^{3}$.

Dos años antes de su muerte, el estado de salud de Carlos II era deplorable. Su debilidad se había acentuado, al punto de no poder permanecer más de una 0 dos horas en pie. A esto se agregaba la presencia de edema generalizado, el cual le afectaba incluso la lengua, impidiéndole hablar. Transitó entre la juventud y senectud aceleradamente, estando su rutina diaria marcada por una triste sucesión de síntomas: edema, fatiga, decaimiento, ataques epilépticos, diarreas y fiebre. Falleció un $1^{\underline{0}}$ de noviembre, tras permanecer dos días en coma producto de una diamea severa, concluyendo con él la dinastía de los Habsburgo. Su autopsia reveló aun corazón del tamaño de un grano de pimienta, los pulmones comoídos, los intestinos putrefactos y gangrenosos, en el rinón tres grandes cálculos, un solo testículo, negro como el carbón y la cabeza llena de agua»? 
Carlos II heredó el trono a su sobrino nieto, Felipe de Borbón, Duque de Anjou. La medida fue ampliamente resistida por las casas reales de Inglaterra, Holanda y Austria, pues implicaba la unión del trono español y francés bajo la misma dinastía, los Borbón. Esta diferencia se resolvió luego de un largo y sangriento conflicto conocido como la Guerra de Sucesión Española (1702-1713), tras la cual se confirmó en España la regencia del Duque de Anjou (nieto de Luis XIV, el Rey Sol) bajo el nombre de Felipe V, iniciándose así la dinastía borbónica española, vigente en la actualidad ${ }^{1}$.

\section{ASPECTOS MÉDICOS}

Resulta todo un desafío plantear una unidad diagnóstica en torno al singular conjunto de síntomas y signos que el rey Carlos II presentó a lo largo de su vida, especialmente si se analizan referencias distantes en el tiempo. Gregorio Marañón (1887-1960), destacado endocrinólogo e historiador español, propuso el diagnóstico de panhipopituitarismo con progeria, sin embargo, de forma casi unánime los historiadores han imputado sus defectos más bien a la consanguinidad de su linaje. Para historiadores como Lynch, «arlos II fue la última, la más degenerada y la más patética víctima de la endogamia de los Habsburgo ${ }^{5}$. Basta

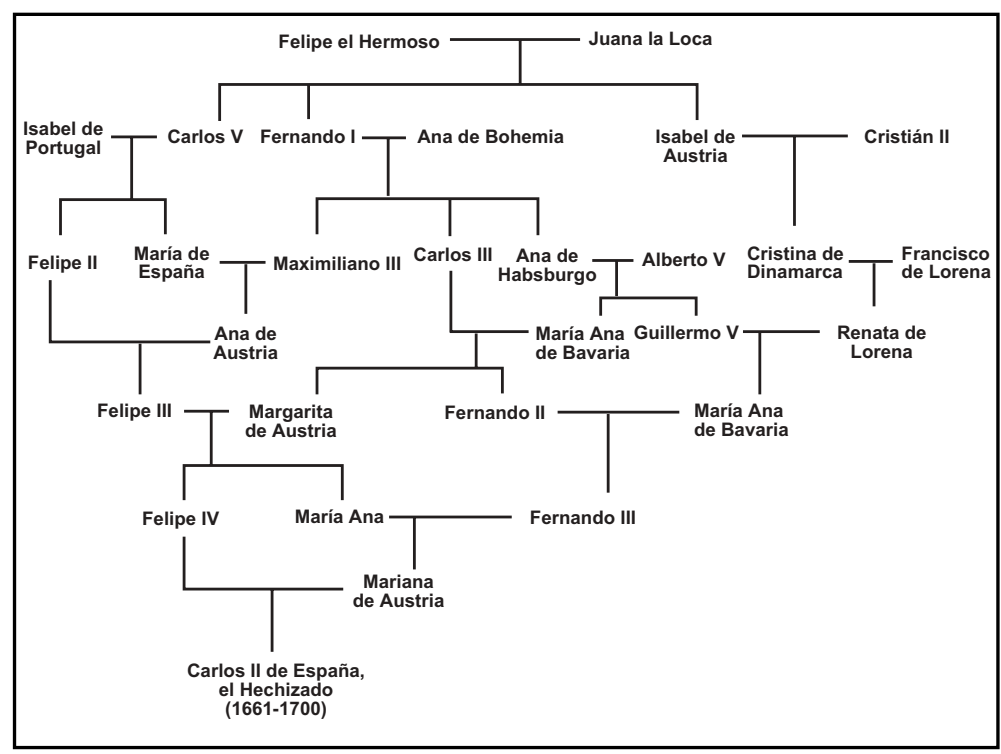

Figura 2. Genograma de Carlos II de España, el Hechizado» Adaptación del original "The ancestry of King Charles II of Spain» disponible en www.answers.com. con observar con detención su genograma; en palabras de García-Argüelles, en el tronco familiar figuran repetidos los nombres de Felipe el Hermoso y Juana la Loca ocho veces; los de Femando I y Ana de Bohemia, nueve; Carlos V e Isabel de Portugal, cuatro; Felipe III y Margarita de Austria son, a la vez, sus abuelos y bisabuelos. Su padre estaba casado con una hija de su hermana, por lo que, a la vez, era tío segundo de su hijo y su madre resulta ser prima de su

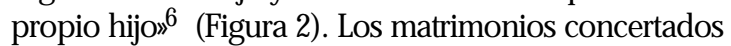
entre los miembros de distintas familias reales europeas han sido una constante a través de los siglos, favoreciendo la endogamia y, por consiguiente, la expresión fenotípica de trastomos genéticos poco frecuentes en la población. Ejemplo de ello es la ocurrencia de hemofilia entre los varones descendientes de la Reina Victoria de Inglaterra (1819-1901), cuya familia se expandió generosamente en las diversas casas reales europeas.

La conjunción de elementos orgánicos, psiquiátricos y conductuales en la persona del Rey Carlos II motivaron a otros investigadores a plantear la hipótesis de que éste presentara una cromosomopatía, específicamente el síndrome de Klinefelter (SK) ${ }^{3}$. Descrito en 1942 por el Dr. Hamy Klinefelter ${ }^{7}$, éste comesponde a la aneuploidía de los cromosomas sexuales más común de la especie humana y la causa más frecuente de hipogonadismo e infertilidad masculina, afectando entre 1:500 y 1:1.000 varones. Los individuos afectados tienen uno 0 más cromosomas $\mathrm{X}$ extra, siendo el complemento 47,XXY el más frecuente (80\%). Sus caracteństicas fenotípicas más comunes incluyen infertilidad, niveles inadecuados de testosterona, hipogonadismo, hipogenitalismo, ginecomastia, trastomos conductuales y aspecto eunucoide (talla alta, extremidades largas, escaso vello facial y distribución de vello de tipo femenino). Otras anomalías asociadas al SK son criptorquidia, hipospadias y escoliosis, así como diabetes y bronquitis crónica en la adultez ${ }^{8,9}$. El SK puede pasar inadvertido durante años, debido a que no existe un fenotipo caracteństico en etapa preadolescente. Durante la infancia, los pacientes 
pueden presentar trastomos del desarmollo (ej: retraso en la adquisición de habilidades motoras y lingüísticas) $\mathrm{y}$ en algunos individuos puede cursar con retardo mental, generalmente leve. El hipogonadismo no se hace evidente sino hasta la pubertad o la adultez, siendo algunos varones diagnosticados en el contexto de una evaluación por infertilidad ${ }^{8,9}$. Se han reportado otros patrones cromosómicos en el SK, por ejemplo, mosaicismo $46, \mathrm{XY} / 47, \mathrm{XXY}^{10}$, es decir, la presencia simultánea de dos líneas celulares diferentes en un mismo individuo. Los adultos portadores de mosaicismo XY/XXYY presentan menor frecuencia y severidad de signos clínicos; tal pudo ser el caso de Carlos II, quien no presentaba algunos elementos frecuentemente encontrados en el SK, por ejemplo, ginecomastia y estatura alta 8,9 ; sin embargo, la severidad del cuadro clínico del monarca y la existencia de signos y síntomas ajenos al SK reducen la posibilidad que efectivamente haya presentado esta aneuploidía cromosómica. De gran interés seńa estudiar fragmentos de $\mathrm{ADN}$ de Carlos II utilizando técnicas de biología molecular contemporáneas, investigación que aportańa objetividad a los diagnósticos presuntivos.

Por su parte, el síndrome edematoso que el monarca desarmolló hacia el final de su vida puede haber sido consecuencia de una nefropatía consecutiva a infecciones recurrentes por cálculos renales, tal cual evidenciara su autopsia ${ }^{3}$, o bien producto de una insuficiencia cardíaca progresiva; en palabras de Geelen, su médico flamenco, sal rey se le para el

\section{REFERENCIAS}

1. Spain under the Habsburgs. Charles II. Disponible en www.britannica.com (Consultado el 21/06/07).

2. Charies II OF Spain. Disponible en http:// www.answers.com (Consultado el 21/06/07).

3. САSTшо A. Carlos II: el fin de una dinastía enferma. Disponible en http://www.arturosonia.com (Consultado el 21/06/07).

4. Hodge GP. A medical history of the Spanish Habsburgs. As traced in portraits. JAMA 1977; 238:1169-74.

5. BITtERnut P. Carlos II. Disponible en http://www.lenguasdefuego. net/CarlosII (Consultado el 21/06/07).

6. Rвот L Biografías: Carlos II. El monarca incapaz. Disponible en http://www.cliorevista.orange.es/clio/ reportajes/ $1210 \mathrm{l} . \mathrm{html}$ (Consultado el 01/08/07).

7. KunEFELTER HR, REIFFENSTEIN EC, Aibright F. Syndrome corazón (sic) y empeora visiblemente. Se le hinchan el vientre, las piemas y la cara... le han hallado todas las entrañas... y el corazón tan consumido y seco ${ }^{\text {. }}$. Rico-Avello plantea una hipótesis altemativa. En su opinión, las repetidas diameas, los cólicos y los pujos dolorosos, los vómitos, la acusada anorexia y el adelgazamiento que caracterizaron su enfermedad final pudieron ser la manifestación de una enterocolitis crónica, consecutiva a la generalización de un proceso tuberculoso ${ }^{6}$. Hasta el día de hoy, su diagnóstico definitivo sigue siendo un misterio.

Finalmente, la historia del Rey Carlos II es un ejemplo del profundo arraigo que tenía tanto en la conciencia de la sociedad castellana del siglo XVII como en los médicos cortesanos la creencia en fenómenos de tipo sobrenatural, los cuales tenían un rol explicatorio de enfermedades, en especial aquellas que cursaban con cuadros clínicos especiales. La hipótesis del hechizo proferido contra el monarca ofrecía una explicación satisfactoria para las mentes de la época, quienes observaban atónitos los estigmas físicos y el extraño comportamiento que exhibía su monarca. La ilustración y los progresos de la ciencia no lograron desterrar del todo tales creencias, siendo transmitidas generacionalmente, estando plenamente vigentes en diversas sociedades alrededor del mundo hasta el día de hoy; ejemplo de ello es la creencia en el mal de ojo», tan amaigada en nuestro país, en pleno siglo XXI.

characterized by gynecomastia, aspermatogenesis without a-Leydism, and increased excretion of follicle-stimulating homone. J Clin Endocrinol 1942; 2: 615-27.

8. Sмттн S. Index of suspicion. Case 1. Diagnosis: Klinefelter syndrome. Pediatr Rev 1992; 13: 435-7.

9. Duong M, Dinoulos Jg, Gupta A, Bryk T, Saps M, Di LoRENZO C ET AL Index of suspicion. Case 5. Diagnosis: Klinefelter syndrome. Pediatr Rev 2005; 26: 23-33.

10. Eussartou V, Christopoulou S, Karadimas C, Pihos I, KanaKa-GantenBeIN, KapRanos N et al. Rare XXY/XX mosaicism in a phenotypic male with Klinefelter syndrome: case report. Eur J Med Genet 2006; 49: 331-7.

11. LópEz ALonso A. Carlos II, el rey al que todos creyeron hechizado. Disponible en http:// www.edicionesirreverentes.com/Anoticias/datos/carlos3.htm (consultado el 01/08/07). 